\title{
DYNAMICAL STUDY OF A1689 FROM WIDE-FIELD VLT/VIMOS SPECTROSCOPY: MASS PROFILE, CONCENTRATION PARAMETER, AND VELOCITY ANISOTROPY
}

\author{
Doron Lemze ${ }^{1}$, Tom Broadhurst ${ }^{1}$, Yoel Rephaeli ${ }^{1,2}$, Rennan Barkana $^{1,3,4}$, and Keitchi Umetsu ${ }^{5,6}$ \\ ${ }^{1}$ School of Physics and Astronomy, Tel Aviv University, Tel Aviv, 69978, Israel; doronl@wise.tau.ac.il \\ ${ }^{2}$ Center for Astrophysics and Space Sciences, University of California, San Diego, CA, USA \\ ${ }^{3}$ Institute for Cosmic Ray Research, University of Tokyo, Kashiwa 277-8582, Japan \\ ${ }^{4}$ Division of Physics, Mathematics and Astronomy, California Institute of Technology, Mail Code 130-33, Pasadena, CA 91125, USA \\ 5 Institute of Astronomy and Astrophysics, Academia Sinica, P.O. Box 23-141, Taipei 106, Taiwan \\ ${ }^{6}$ Leung Center for Cosmology and Particle Astrophysics, National Taiwan University, Taiwan \\ Received 2009 January 7; accepted 2009 June 17; published 2009 July 31
}

\begin{abstract}
We examine the dynamics structure of the rich cluster A1689, combining VLT/VIMOS spectroscopy with Subaru/ Suprime-Cam imaging. The radial velocity distribution of $\sim 500$ cluster members is bounded by a pair of clearly defined velocity caustics, with a maximum amplitude of $\sim|4000| \mathrm{km} \mathrm{s}^{-1}$ at $\simeq 300 \mathrm{~h}^{-1} \mathrm{kpc}$, beyond which the amplitude steadily declines, approaching zero velocity at a limiting radius of $\sim 2 h^{-1} \mathrm{Mpc}$. We derive the three-dimensional velocity anisotropy and galaxy number density profiles using a model-independent method to solve the Jeans equation, simultaneously incorporating the observed velocity dispersion profile, the galaxy counts from deep Subaru imaging, and our previously derived cluster mass profile from a joint lensing and Xray analysis. The velocity anisotropy is found to be predominantly radial at large radius, becoming increasingly tangential towards the center, in accord with expectations. We also analyze the galaxy data independently of our previous analysis using two different methods: The first is based on a solution of the Jeans equation assuming an Navarro-Frenk-White (NFW) form for the mass distribution, whereas in the second method the caustic amplitude is used to determine the escape velocity. The cluster virial mass derived by both of these dynamical methods is in good agreement with results from our earlier lensing and X-ray analysis. We also confirm the high NFW concentration parameter, with results from both methods combined to yield $c_{\text {vir }}>13$ $(1 \sigma)$. The inferred virial radius is consistent with the limiting radius where the caustics approach zero velocity and where the counts of cluster members drop off, suggesting that infall onto A1689 is currently not significant.
\end{abstract}

Key words: galaxies: clusters: individual (A1689) - galaxies: kinematics and dynamics - methods: data analysis

Online-only material: color figures

\section{INTRODUCTION}

Clusters of galaxies display a wide range of distinct observational phenomena, providing detailed physical information of central importance to cosmology. Recent observations have resulted in an impressive consistency with the predictions of the standard $\Lambda$ CDM model (Tegmark et al. 2004; Spergel et al. 2007 and references therein). In this model, a cosmological constant dominates the cosmic energy budget today, but galaxies and other structures were assembled earlier, primarily out of cold dark matter (CDM). While the model successfully matches observations of the primary anisotropy of the cosmic microwave background and the large-scale structure in galaxy surveys, it is also important to test its validity on smaller scales. The abundance and structure of nonlinear objects are sensitive probes of the properties of dark matter (DM) and of the primordial density fluctuation field. Gas cooling and various feedback mechanisms complicate the interpretation of the DM distribution in galaxies (e.g., Bower et al. 2006). However, for clusters most of the gas is observed to be too hot and rarefied to cool efficiently and is thus expected to be in hydrodynamical equilibrium at the virial temperature, and therefore to have only a small influence on the nonbaryonic mass distribution (outside the inner core). Hence it is reasonable to suppose that constraints on the mass profile of a cluster probe the dominant DM.

Interaction of a cluster with another cluster or group of galaxies is commonly indicated by the presence of shock fronts, seen in high quality X-ray observations. In the case of the "bullet cluster," a cone-shaped shock front is visible indicating that two clusters have passed through each other, with an obvious merger of the intracluster (IC) gas of the two clusters, but the galaxies and the lensing mass distribution are largely intact. This directly implies that the DM is collisionless like the galaxies (Markevitch et al. 2002; Clowe et al. 2004; Bradač et al. 2006), supporting the simplest possibility that DM interacts only via gravity.

For relaxed clusters, knowledge of the mass profile can provide crucial information regarding the nature of DM and the thermal history of IC gas. Precise measurements have been made by dynamical analysis of galaxy velocities, via hydrostatic analysis of X-ray observations, or directly via lensing. Detailed measurements of the X-ray spectrum and intensity profile yield the distribution of total mass through solving the equation of hydrostatic equilibrium. In practice the temperature measurements are often made uncertain by significant complications such as the likely multiphase nature of gas in equilibrium. We have recently developed a model-independent joint lensing/X-ray analysis (Lemze et al. 2008, hereafter L08) to examine the consistency of the X-ray temperature and surface brightness profiles with the lensing data, finding that the cluster's spectrally measured temperature profile is systematically $\sim 30 \%-40 \%$ lower than deduced from solving the equation of hydrostatic equilibrium with the precisely measured lensing mass profile. This may reflect in part the ambiguity in deriving three-dimensional temperatures from projected X-ray data, since a given line of 
sight will in general intersect a range of gas temperatures if the gas is not strictly isothermal (Mazzotta et al. 2004; Vikhlinin 2006). It is also conceivable that the gas is not strictly single phase but may contain small-scale structure, including relatively dense cooler clouds as found in detailed simulations (Kawahara et al. 2007), which may lead to a significant downward bias in observed temperature estimates.

The velocity dispersion profile of clusters has long been used to estimate the galaxy dynamics and the cluster mass profile via the Jeans equation, when sufficient redshift information is available. This technique was originally applied to several well studied nearby clusters (Fuchs \& Materne 1982; Sharples et al. 1988), and more recently to the results of dedicated surveys (Carlberg et al. 1997; Katgert et al. 2004; Biviano \& Katgert 2004; Hwang \& Lee 2008). In these studies the expected velocity anisotropy complicates the interpretation, as orbits are not expected to be isotropic but to become predominantly radial towards the cluster's virial radius. More recently, with the availability of larger samples of redshift measurements, it has been recognized that relaxed clusters should have sufficiently well defined velocity "caustics," providing an independent means of deriving cluster mass profiles; this has been applied to several clusters and compared with lensing-based masses (Geller et al. 1999; Rines et al. 2003; Diaferio et al. 2005). Joint studies of galaxy dynamics and lensing in the central cluster region are also providing detailed mappings of the central mass distributions of massive clusters, where the velocity dispersion profile of the central galaxy can be compared with the analysis of multiply imaged sources (Sand et al. 2008).

Lensing work is now able to measure cluster mass profiles with sufficient precision to usefully test the distinctive prediction of a relatively shallow mass profile for halos dominated by CDM (Navarro et al. 1997, hereafter NFW; Hennawi et al. 2007; Duffy et al. 2008). Combined weak and strong lensing measurements have shown that the continuously steepening form of the NFW profile is a reasonable description for the mass profiles of three carefully studied clusters (Kneib et al. 2003; Gavazzi et al. 2003; Broadhurst et al. 2005 a, hereafter B05a), although with surprisingly high values derived for the profile's characteristic concentration parameter in each case (B05a). More recently, the concordance $\Lambda \mathrm{CDM}$ model has been examined with increasing (though still relatively small) samples of clusters, indicating that the concentrations derived are significantly higher than predicted over a wide range of cluster mass, after a statistical correction for lensing-induced biases. This is seen both in terms of the size of the Einstein radius (Broadhurst \& Barkana 2008) and the weak lensing profiles of several well known clusters (Broadhurst et al. 2008). This question has also been examined recently by stacking the lensing signal of cluster samples identified in the SDSS survey. A wide dispersion in derived concentrations is found, with an average value in better agreement with the predicted relation (Mandelbaum et al. 2008). Here it is crucial that reliance on photometric redshifts does not result in the misclassification of cluster members as background galaxies, which would artificially decrease the central lensing signal thereby lowering measured concentrations.

Here we concentrate on the internal dynamics of A1689, one of the best studied clusters, using a relatively large redshift survey from the VLT/VIMOS wide-field instrument. This cluster is attractive as it is very massive and appears to be relaxed, with a highly symmetric X-ray morphology (L08; Riemer-Sorensen et al. 2009, see their Figure 1), the centroid of which coincides both with the cD galaxy and the center of mass derived from lensing (Xue \& Wu 2002; L08). Only a low level of substructure is visible in detailed lensing and X-ray maps (B05a; Broadhurst et al. 2005b; L08; Umetsu \& Broadhurst 2008), though Andersson \& Madejski (2004) claim an indication for a small deviation from a relaxed state. Earlier dynamical analyses were made by den Hartog \& Katgert (1996), Czoske (2004), and Łokas et al. (2006). In our earlier work we found that the cluster has a relatively high concentration parameter, which makes this a very interesting target to explore with independent dynamical means. We also make use of the extensive VLT/VIMOS data to explore galaxy dynamical properties in unprecedented detail, including in particular the velocity anisotropy profile, which has been difficult to determine from previous observations (Benatov et al. 2006).

This paper is organized as follows. In Section 2 we describe the data, from which we derive the radial profile of the projected surface density (Section 2.1), and the projected velocity distribution and radial profile of the velocity caustics (Section 2.2). In Section 3 we detail our method of using the Jeans equation together with previous knowledge of the cluster mass profile to determine the three-dimensional radial profiles of galaxy velocity dispersion and anisotropy, with results given in Section 4. In Section 5 we independently determine the cluster mass profile from the data on galaxy dynamics, using the caustics as a measure of the escape velocity from the cluster (Section 5.1), or fitting an NFW profile to the dynamical data (Section 5.2). We compare in Section 6 various definitions of the cluster boundary, based on the radial profiles of the cluster mass, galaxy density, or caustics, and end with a discussion in Section 7.

\section{DATA ANALYSIS}

We use several different types of data in this paper: galaxy positions and velocities, gravitational lensing, both strong and weak, and X-ray emission and spectroscopy. Previously we have made joint use of the same lensing and X-ray data to derive a cluster mass profile by a model-independent joint analysis as described in L08. Here we extend this work and analyze independent dynamical information on the galaxy velocities and also the number density profile of cluster members. In this section we explain in detail our analysis of the latter two data sets.

\subsection{Galaxy Surface Number Density}

Establishing the form of the projected profile of cluster member galaxies is essential for fully employing the Jeans equation (Binney \& Tremaine 1987; see Section 3), which allows for a spatial distribution of galaxies that need not follow the dominant DM. Measurement of the projected galaxy distribution requires subtraction of the background and foreground field galaxy populations. This must be achieved with accurate multicolor photometry as spectroscopy is usually limited to small samples and does not extend faint enough to include the majority of cluster members.

We used Subaru photometry in the $V$ and $I$ bands. The data are complete to a depth of $I_{A B}=26.5$, reaching eight magnitudes below $L^{*}$ of the luminosity function. We selected a color region based on a color-magnitude relation analysis, which comprises 17474 galaxies. This color region ranges from the red side of the E/SO cluster sequence to a blue boundary chosen so that the sample extends sufficiently blueward to include most $(\sim 85 \%)$ 


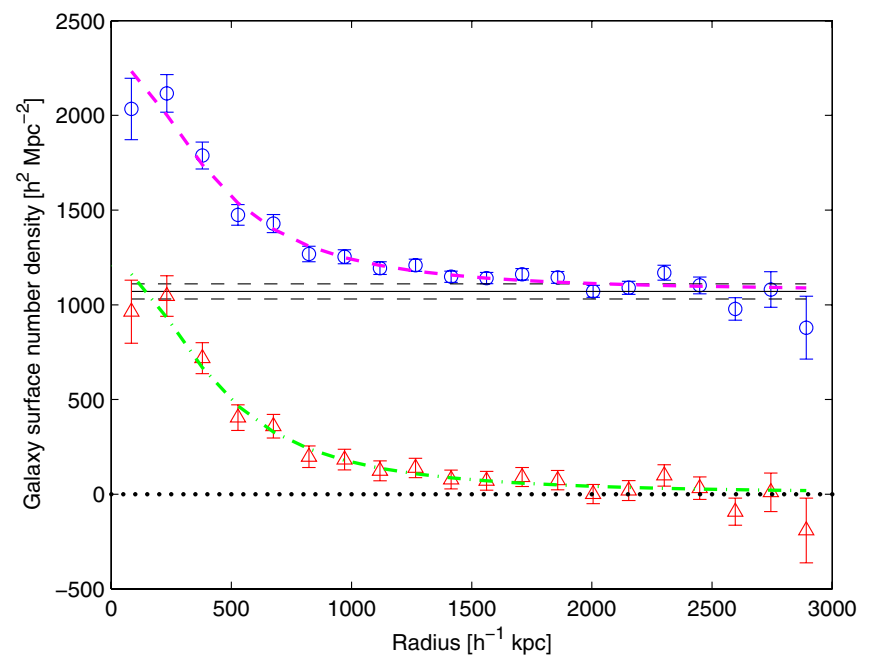

Figure 1. Galaxy surface number density. We show the total (blue circles), the cluster contribution (red triangles), and the background value (solid horizontal line) and its uncertainty (dashed lines). All uncertainties are $1 \sigma$. We also show the core profile that best fits the cluster galaxy surface number density (dashdotted curve), and the corresponding fit to the total galaxy surface number density (dashed curve).

(A color version of this figure is available in the online journal.)

of the cluster members as described in Medezinski et al. (2007, hereafter M07, Figure 1). M07 established that this color region contains the majority of cluster members in addition to some background galaxies, by examining the weak lensing signal. In this color region, which includes the E/SO sequence and bluer objects (including some background galaxies) the lensing signal was found to be significantly lower than the true background signal as measured for red background galaxies. This is because (unlensed) cluster members dilute the weak lensing signal of the background, allowing us to identify the region of color space occupied by cluster members.

We derived the projected galaxy distribution of cluster members from the above color-selected galaxy sample based on fitting the radial distribution. Galaxies were first radially binned into annuli and the surface number density in each annulus was determined; note that at large radii only part of each annulus was covered by the detector. Assuming Poissonian $(\sqrt{N})$ errors, the galaxy surface number density was modeled as that due to the cluster plus a background galaxy surface number density, with the latter assumed uniform. Thus, we effectively neglected fluctuations in the background galaxy surface number density on scales smaller the cluster size (about $22^{\prime}$ at $z=0.183$ ). There could be such fluctuations at some level due to correlated structures (such as filaments) along the line of sight to the cluster. Galaxies in these structures could mistakenly be included as cluster members, a possibility that we assess below.

Figure 1 shows that the radial profile of the above colorselected galaxy sample is well fitted with a uniform background plus a general cored profile to represent the cluster galaxy surface density profile:

$$
\Sigma_{\mathrm{tot}}=\Sigma_{\mathrm{gal}}+C_{\mathrm{bg}}=\frac{\Sigma_{0}}{\left[1+\left(r / r_{c}\right)^{2}\right]^{p}}+C_{\mathrm{bg}}
$$

where $\Sigma_{\text {tot }}$ is the total galaxy surface number density, $\Sigma_{0}$, $r_{c}$, and $p$ are the three parameters of the cored profile, and $C_{\mathrm{bg}}$ is the background density. The resulting fit is good, $\chi^{2} /$ dof $=19.2 / 16$. Using a larger number of bins than $N=20$ improves the reduced $\chi^{2}$, e.g., $N=50$ and $N=100$ give

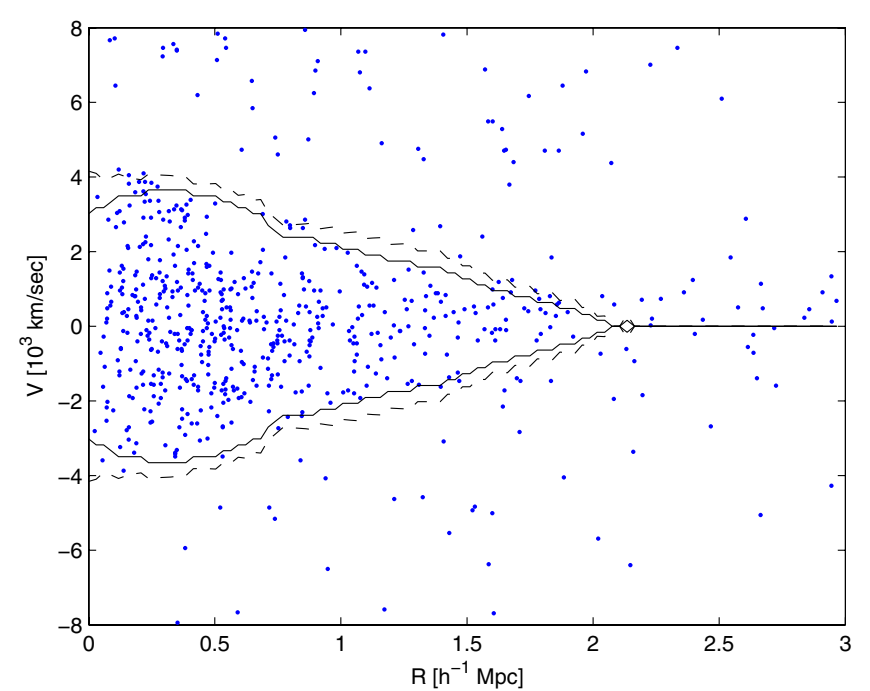

Figure 2. Velocity-space diagram of A1689. The caustics are shown along with $1 \sigma$ error bars (shown only on the outer side of the caustics for clarity).

(A color version of this figure is available in the online journal.)

$\chi^{2} /$ dof $=36 / 46$ and $\chi^{2} /$ dof $=96 / 96$, respectively. The level of the background is stable and does not depend on the number of bins, e.g., for $N=20,50$, and 100 we find $C=1071 \pm 40$, $1064 \pm 42$, and $1063 \pm 41$, respectively. We settled on just 20 bins, since this made it easier to combine the surface density data set with the projected velocity dispersion data set, which is inferior in terms of signal to noise per radial bin (see below). With 20 bins, we found best-fit values of $\Sigma_{0}=1200 \pm$ $140 h^{2} \mathrm{Mpc}^{-2}, r_{c}=450 \pm 150 h^{-1} \mathrm{kpc}$, and $p=0.74 \pm 0.30$.

\subsection{Projected Velocity Dispersion}

To measure the mass profile of galaxy clusters using galaxy motions it is necessary to obtain precise velocity measurements for a statistically large sample of galaxies. The data used here are part of an extensive multi-object spectroscopy survey carried out with the VIMOS spectrograph on the VLT (Czoske 2004); for observational details, see Czoske (2004). This data set constitutes 1469 objects with reliable spectroscopic redshifts, a major advance over previous surveys of A1689. Note though that we did not try to find the galaxy surface density profile from the projected velocity data set, since the data set used in the previous section contains a much larger number of galaxies.

We analyzed the spectroscopic sample by first defining cluster membership using the velocity "caustics," which are clearly visible for this cluster in the form of a boundary which varies with radius, peaking at around $\pm 4000 \mathrm{~km} \mathrm{~s}^{-1}$ at $\sim 300 \mathrm{~h}^{-1}$ $\mathrm{kpc}$ and declining steadily at larger radius (see Figure 2). The caustics are related to the escape velocity from the cluster and thus provide a tangible physical basis by which we can separate cluster members from foreground and background galaxies. Defining membership is especially important for massive clusters like A1689, since they have a relatively wide internal velocity spread and they extend to large radii; this increases the chance for interlopers, which can have a large effect on the derived projected velocity dispersion, especially at large radii where the number density of cluster members is low. Wojtak \& Łokas (2007) showed that without the proper removal of interlopers the inferred parameters of the mass distribution in the cluster are strongly biased towards higher mass and lower concentration. See also Wojtak et al. (2007) for interesting comparison between different methods of interlopers removal, 
which unfortunately do not include the D99 caustics approach used in this paper.

To define the caustics we employed the technique pioneered by Diaferio (1999, hereafter D99) based on a multidimensional adaptive kernel method (Silverman 1986; Pisani 1993; Pisani 1996). A summary of the application of this technique can be found in Diaferio et al. (2005); it has previously been applied to several clusters (Reisenegger et al. 2000; Biviano \& Girardi 2003; Diaferio et al. 2005; Rines \& Diaferio 2006). Briefly, a redshift-space diagram is constructed, i.e., the line of sight velocity $v$ is plotted versus the projected distance $R$ from the cluster center, and for a well defined cluster these points should be distributed in a characteristic "trumpet" shape, the boundaries of which are termed caustics (Kaiser 1987; Regos \& Geller 1989). The D99 procedure locates the caustics and determines the radial dependence of their amplitude (in units of velocity), which is related to the escape velocity and thus depends on the mass profile. Galaxies that are inside the caustics can then be considered to be cluster members. Of course, some of these galaxies might be interlopers, but their number is typically a few percent and has little effect on dynamical analyses (A. Diaferio 2008, private communication).

To apply this procedure we determined the threshold $\kappa$ that defines the caustic location through $f_{q}(R, v)=\kappa$ (D99). Here $f_{q}(R, v)$ is the galaxy density distribution in the redshift-space diagram, smoothed with an adaptive kernel, and $v$ is the peculiar velocity (measured with respect to the cluster's mean redshift). The parameter $q$ sets the scaling between the quantities $R$ and $v$ within the smoothing procedure. We used $q=27$, close to the value usually used, 25 (D99; Rines et al. 2003). We note that different values of $q$ in the 10-50 range have little effect on the results (D99).

The parameter $\kappa$ was chosen by minimizing the quantity $S(\kappa,\langle R\rangle)=\left|\left\langle v_{\text {esc }}^{2}\right\rangle_{\kappa,\langle R\rangle}-4\left\langle v^{2}\right\rangle\right|^{2}$, where $\left\langle v_{\text {esc }}^{2}\right\rangle_{\kappa,\langle R\rangle}=$ $\int_{0}^{\langle R\rangle} A^{2}(R) \phi(R) d R / \int_{0}^{\langle R\rangle} \phi(R) d R$ is the mean value of the square of the caustic amplitude $A(R)$ within $\langle R\rangle$ (the mean projected radius of the cluster members), $\phi(R)=\int f_{q}(R, v) d v$, and $\left\langle v^{2}\right\rangle$ is the one-dimensional velocity dispersion of the cluster members. The uncertainty of $A$ is proportional to the inverse of the galaxy number density within the caustics, and was estimated as $\delta A(R) / A(R)=\kappa / \max \left\{f_{q}(R, v)\right\}$, where the maximum is found along the $v$-axis at each $R$ (D99).

Figure 2 shows the velocity-space diagram and radial profile of the derived velocity caustics. There are a total of $476_{-43}^{+27}$ galaxies identified as cluster members lying between these caustics. The velocity-space diagram clearly looks qualitatively different inside and outside the caustics, indicating that the caustic-finding procedure has identified a physically meaningful boundary. The density of the galaxies that lie between the caustics shows a smooth decline with distance from the center, indicating that the cluster has a simple monolithic dynamical structure. The radius at which the caustics meet at zero velocity represents an effective maximum radius for the cluster, which physically includes cluster galaxies both within the virial radius and others that may be currently infalling onto the cluster (see the discussion in Section 7). Note that the two caustics are by definition symmetric about $v=0$ in this method, which Figure 2 clearly shows is a valid assumption for this cluster.

Having defined cluster membership based on the location of the caustics, we can estimate the projected velocity dispersion profile restricted to cluster members. We divided the cluster members into velocity bins of size $600 \mathrm{~km} \mathrm{~s}^{-1}$, suffi-
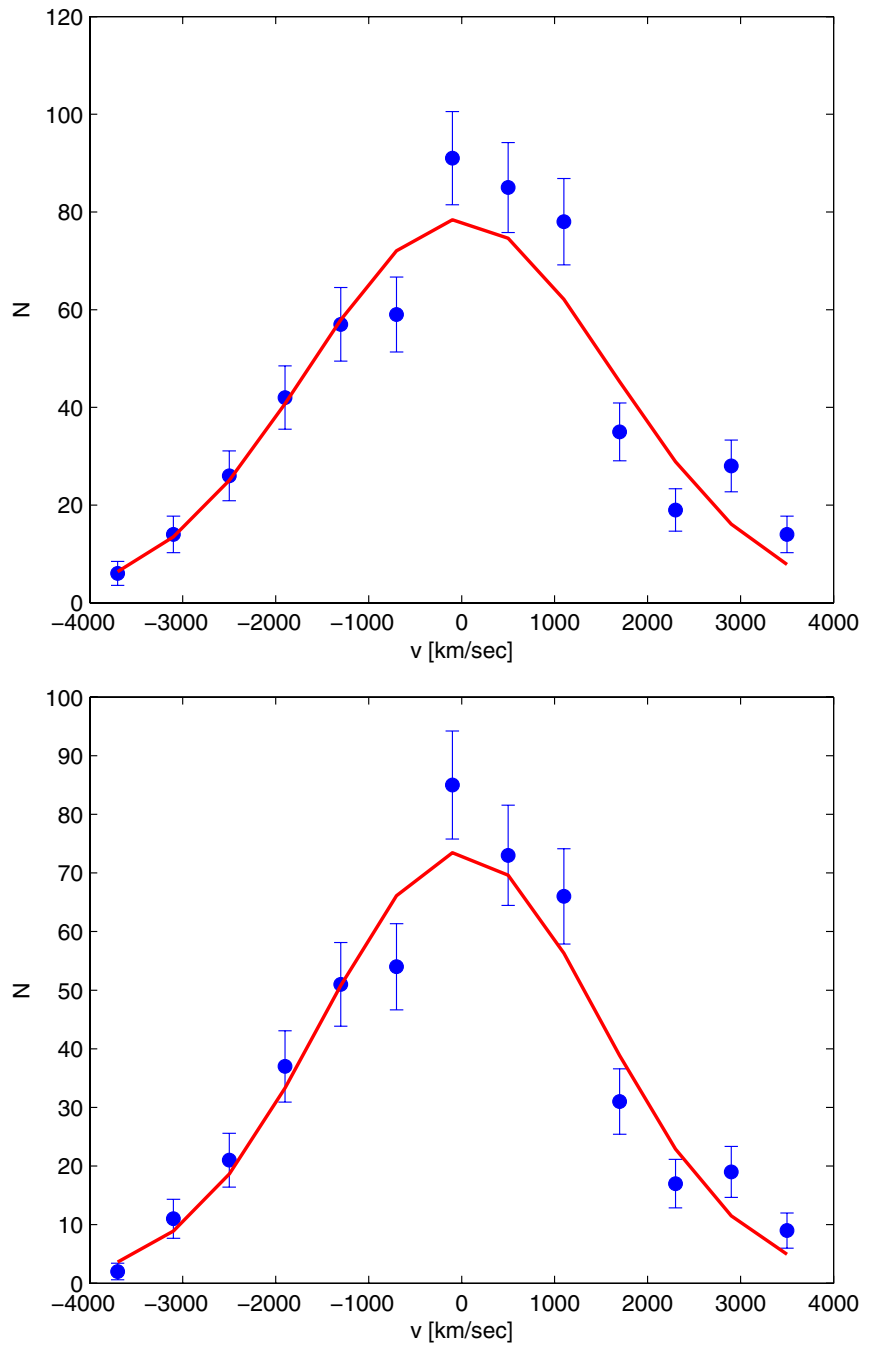

Figure 3. Observed histogram of galaxy velocities and Gaussian fits. Top panel: all galaxies; the fit yielded $\chi_{r}^{2}=2.5$ with derived parameters $z_{\text {cluster }}=$ $0.185 \pm 0.0003$ and $\sigma_{p}=(1.75 \pm 0.08) \times 10^{3} \mathrm{~km} \mathrm{~s}^{-1}$. Bottom panel: only cluster members, whose identification is based on the caustics; the fit yielded $\chi_{r}^{2}=1.7$ with $\sigma_{p}=(1.40 \pm 0.06) \times 10^{3} \mathrm{~km} \mathrm{~s}^{-1}$.

(A color version of this figure is available in the online journal.)

ciently large compared to the redshift measurement uncertainty of $\sim 200 \mathrm{~km} \mathrm{~s}^{-1}$. Different binning gave very similar results. We fitted a Gaussian to the velocity histogram to calculate the overall projected velocity dispersion (Figure 3 ); it provided a reasonable fit with a reduced $\chi^{2}$ (hereafter $\chi_{r}^{2}$ ) of 1.7 per degree of freedom. If we ignore the caustics and simply bin all galaxies within the maximal velocity range $|v|<4000 \mathrm{~km} \mathrm{~s}^{-1}$, the fit to a Gaussian is significantly worse, $\chi_{r}^{2}=2.5$, with a more asymmetric distribution and a somewhat larger velocity dispersion. It is important to mention that we did not use the results of the Gaussian fit in our analysis. We show this fit to argue in favor of the real physical meaning of the caustics as we have measured them, and demonstrate that without excluding unrelated interlopers with large velocities, dynamically derived quantities of the cluster may be significantly skewed, particularly at large projected radii. Note that we fitted a Gaussian for simplicity, although the real distribution may be slightly non-Gaussian (see, e.g., Kazantzidis et al. 2004; Sanchis et al. 2004; Diemand et al. 2004; Wojtak et al. 2005).

We next estimated the radial profile of the projected velocity dispersion from our sample of cluster members identified within 
the caustic boundaries. We divided the data into 10 radial bins, so that in each bin there were at least 47 galaxies. The radius assigned to each bin was the mean of the measured radii of galaxies lying within the bin. The projected velocity dispersion was taken to be the standard deviation of the galaxy velocities in the bin. The error in the projected velocity dispersion $\Delta \sigma_{p}(R)$ was calculated as $\left(\Delta \sigma_{p}(R)\right)^{2}=\left(\Delta_{\mathrm{me}} \sigma_{p}(R)\right)^{2}+\left(\Delta_{\mathrm{sa}} \sigma_{p}(R)\right)^{2}+$ $\left(\Delta_{\mathrm{ce}} \sigma_{p}(R)\right)^{2}$, where $\Delta_{\mathrm{me}} \sigma_{p}(R), \Delta_{\mathrm{sa}} \sigma_{p}(R)$, and $\Delta_{\mathrm{ce}} \sigma_{p}(R)$ are the measurement, sample, and cluster membership uncertainties, respectively. The measurement error is $\Delta_{\mathrm{me}} \sigma_{p}(R)=1 / \sigma_{p}(R)$. $d v_{i} /(N(R)-1)$, where $N(R)$ is the number of galaxies in the bin, and $d v_{i}=200 \mathrm{~km} \mathrm{~s}^{-1}$ is the measurement error of the projected velocity of the $i$ th galaxy. The $1 \sigma$ sampling error is $\Delta_{\mathrm{sa}} \sigma_{p \pm}(R) / \sigma_{p}(R)=\left[(1-2 /(9 v) \mp \sqrt{2 /(9 v)})^{-3 / 2}-1\right]$, where this formula is valid for $v \geqslant 30$ with $v=N(R)-1$ the number of degrees of freedom (Danese et al. 1980). $\Delta_{\mathrm{ce}} \sigma_{p}(R)$ is the error in the projected velocity dispersion from errors in estimating which galaxies are cluster members and which are interlopers, arising from the uncertainty in the amplitude of the caustic, $\delta A(R)$. We estimated $\Delta_{\text {ce }} \sigma_{p}(R)$ by calculating the difference of the projected velocity dispersion when the cluster members are defined to lie within the caustic defined by the amplitude $A+\delta A$ instead of $A: \Delta_{\mathrm{ce}} \sigma_{p \pm}(R)=\left|\sigma_{p}(A \pm \delta A)-\sigma_{p}(A)\right|$.

There are two previous published measurements of the projected velocity dispersion for A1689. Łokas et al. (2006) took all galaxies in the NASA Extragalactic Database (NED) with redshifts $z=0.1832 \pm 0.05$ and located at projected distances below $2 \mathrm{Mpc}$ from the cluster center. The redshift data for the galaxies came from different surveys, mainly by Teague et al. (1990), Balogh et al. (2002), and Duc et al. (2002). While the sample of Teague et al. (1990) came from a standard magnitudelimited survey, the selection criteria of those of Balogh et al. (2002), and Duc et al. (2002) were aimed at star-forming galaxies, which may bias the sample towards outer regions with more substructure. This full sample comprised 192 galaxies, which they reduced to 130 after defining a constant velocity cutoff of $3000 \mathrm{~km} \mathrm{~s}^{-1}$, estimated visually, which is substantially smaller than the $4000 \mathrm{~km} \mathrm{~s}^{-1}$ maximum amplitude of our caustics. On this basis they claimed significant substructure in velocity along the line of sight, but there is no evidence for such substructure in our velocity data (Czoske 2004), nor is significant substructure seen in the X-ray emission maps or the lensing-based mass map (L08; Umetsu \& Broadhurst 2008). In an earlier work den Hartog \& Katgert (1996) determined the radial velocity profiles of 72 clusters, including A1689. For A1689 they used the data from Teague et al. (1990) comprising only 63 cluster members. The projected velocity profiles derived in these two papers are compared to the one we derived in Figure 4.

\section{METHODOLOGY}

In this section we present our procedure for exploring the structure of the cluster using the above velocity data and the projected profile of the galaxy distribution derived above. In addition, we make use of the mass distribution that we derived in earlier work from a combined X-ray and lensing analysis (L08). In L08 we combined lensing and X-ray measurements to determine model independent profiles of the total mass and the gas mass. Here we add the data on the galaxy surface number density and the projected velocity dispersion, and relate them via the Jeans equation. From this we obtain the three-dimensional galaxy number density profile and the velocity anisotropy
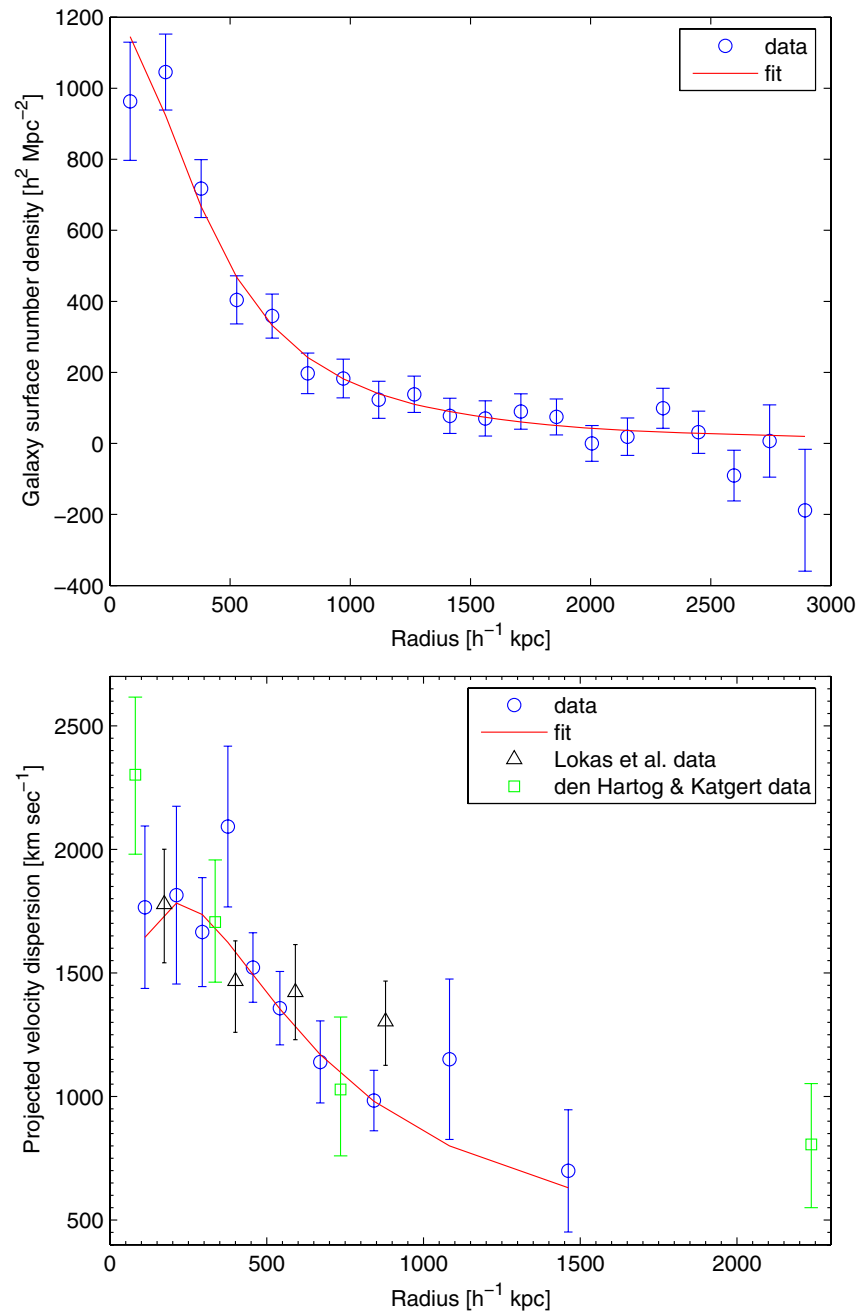

Figure 4. Data and best fits based on analytical profiles. Top panel: the observed profile of galaxy surface number density (blue circles) is compared with our bestfit model profile (red curve). Bottom panel: the observed profile of projected velocity dispersion (blue circles) is compared with our best-fit model profile (red curve). We also show the measurements of Łokas et al. (2006; black triangles) and den Hartog \& Katgert (1996; green squares). In both panels, $1 \sigma$ measurement uncertainties are indicated.

(A color version of this figure is available in the online journal.)

profile. Our work is the first model-free determination of the galaxy velocity anisotropy in a cluster.

In general, we determine the best-fit values of our free parameters by fitting the profiles of galaxy surface number density and projected velocity dispersion simultaneously. We first proceed with simple analytical forms for the relevant profiles, in which we fit for the free parameters of these expressions. We then proceed to a more flexible model-independent approach, similar to that developed in L08. In our model-independent approach, the free parameters are simply the values of the three-dimensional profile of the galaxy number density and velocity anisotropy at several fixed (equally spaced) radii. The radial ranges of these free parameters are set according to the total span of each data set. The values of each quantity (i.e., the galaxy number density or velocity anisotropy) across the cluster are given by linearly interpolating from its values at the fixed radii. Thus, the method only assumes smoothness.

The galaxy number density and velocity dispersion are related by the Jeans equation for a steady-state spherically symmetric 
system,

$$
\frac{d}{d r}\left(n_{\mathrm{gal}}(r) \sigma_{r}^{2}(r)\right)+\frac{2 \beta(r)}{r} n_{\mathrm{gal}}(r) \sigma_{r}^{2}(r)=-\frac{G M(\leqslant r) n_{\mathrm{gal}}(r)}{r^{2}},
$$

where $n_{\text {gal }}(r)$ is the galaxy number density, and $\beta(r)$ is the velocity anisotropy parameter:

$$
\beta(r) \equiv 1-\frac{\sigma_{t}^{2}(r)}{\sigma_{r}^{2}(r)}
$$

where $\sigma_{r}(r)$ and $\sigma_{t}(r)$ are the radial and the tangential components of the velocity dispersion (Binney \& Tremaine 1987). The measured quantities used here are the galaxy surface number density, $\Sigma_{\text {gal }}$,

$$
\Sigma_{\mathrm{gal}}(R)=2 \int_{R}^{\infty} \frac{n_{\mathrm{gal}}(r) r d r}{\sqrt{r^{2}-R^{2}}}
$$

and the observed projected velocity dispersion,

$$
\sigma_{p}^{2}(R)=\frac{2}{\Sigma_{\mathrm{gal}}(R)} \int_{R}^{\infty} \frac{n_{\mathrm{gal}}(r) \sigma_{r}^{2}(r)\left[1-\beta(r) \frac{R^{2}}{r^{2}}\right] r d r}{\sqrt{r^{2}-R^{2}}} .
$$

Given a total cluster mass profile $M(r)$, for any assumed profiles of $n_{\text {gal }}$ and $\beta$ we use the Jeans equation (Equation (2)) to derive the profile of the radial velocity dispersion $\sigma_{r}$. We then use Equations (4) and (5) to compare the model to the two observed data sets, and find the best-fit free parameters. This procedure was first applied using simple analytic forms for the profiles of three-dimensional galaxy number density $n_{\mathrm{gal}}$ and velocity anisotropy parameter $\beta$. The model-independent mass profile found in our earlier work (L08) was used; we also compared in some cases the effect of using its approximation as an NFW profile, with $c_{\mathrm{vir}}=12.2_{-1}^{+0.9}$ and $r_{\mathrm{vir}}=$ $2.14_{-0.29}^{+0.27} h^{-1} \mathrm{Mpc}$ for the concentration parameter and virial radius, respectively (L08). For the galaxy number density profile we assume the $\beta$ model

$$
n_{\mathrm{gal}}(r)=n_{\mathrm{gal}}(0)\left[1+\left(r / r_{s}\right)^{2}\right]^{-\frac{3}{2} p}
$$

where here we use $p$ instead of the standard $\beta$ (which we use for the velocity anisotropy parameter); e.g., the King profile (King 1962) corresponds to $p=1$. N-body simulations for a variety of cosmologies show that the velocity anisotropy has a nearly universal radial profile (Cole \& Lacey 1996). We follow Carlberg et al. (1997) and model it as

$$
\beta=(C+1) \frac{\left(r / r_{\mathrm{vc}}\right)^{2}}{\left(r / r_{\mathrm{vc}}\right)^{2}+1}-C
$$

where $C$ and $r_{\mathrm{vc}}$ (a core radius of the velocity profile) are free parameters. The free parameter $C$ is needed to ensure the freedom for $\beta$ to take on any value in its allowed range, $-\infty<\beta<1$, including circular orbits at small radii. A similar profile is advocated by Girardi et al. (1998) for "case A" clusters, $\beta=\frac{r^{2}}{r^{2}+a^{2}}$, except that we do not constrain the velocities to be isotropic in the center.

When performing model-independent fits, we must extrapolate each quantity beyond the last data point. We extrapolated the anisotropy parameter $\beta$ to be constant, i.e., equal to its value at the last projected velocity dispersion data point. In this way we did not pre-constrain it to equal unity at large radii. We extrapolated the galaxy number density $\propto r^{-3}$, consistent with a King profile and also with the asymptotic behavior of our best-fit $\beta$ model (which is $\propto r^{-3.18 \pm 0.42}$; see Table 1 below).

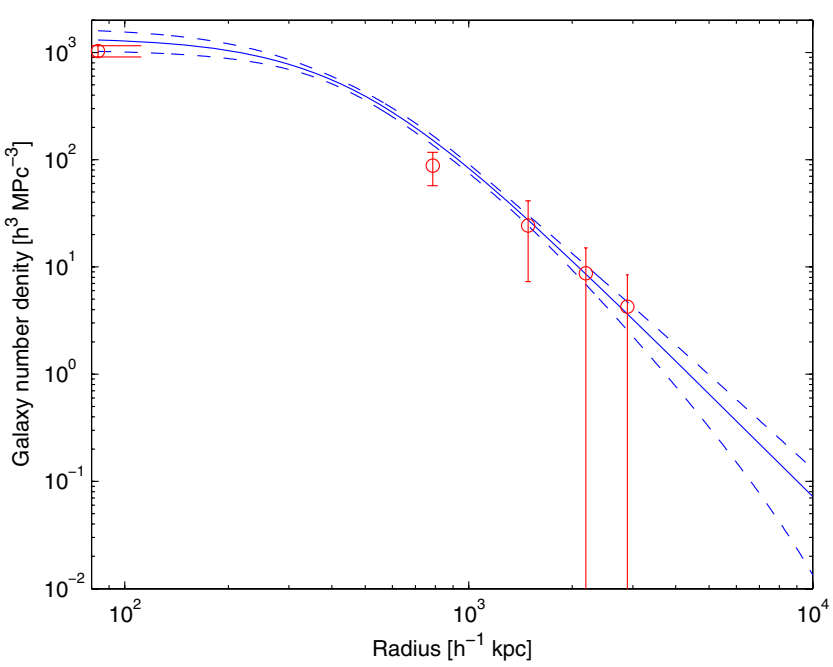

Figure 5. Reconstructed three-dimensional galaxy number density profile. We show the best-fit result and $1 \sigma$ range from fitting a $\beta$ model (solid and dashed curves), where the best-fit model parameters are listed in Table 1. Also shown are the values and $1 \sigma$ errors as derived from the model-independent approach (red circles), where the free parameters were the values of the galaxy number density at five equally (linearly) spaced radii.

(A color version of this figure is available in the online journal.)

Table 1

Best-Fit Parameters for the Galaxy Number Density and Velocity Anisotropy Profiles

\begin{tabular}{lc}
\hline \hline \multicolumn{1}{c}{ Parameter } & Value \\
\hline$n_{\mathrm{gal}}(0)\left(h^{3} \mathrm{Mpc}^{-3}\right)$ & $1380 \pm 320$ \\
$r_{s}\left(h^{-1} \mathrm{kpc}\right)$ & $455 \pm 110$ \\
$p$ & $1.06 \pm 0.14$ \\
$r_{\mathrm{vc}}\left(h^{-1} \mathrm{kpc}\right)$ & $395 \pm 280$ \\
$C$ & $1.8 \pm 2.6$ \\
\hline
\end{tabular}

\section{THE DYNAMICAL PROPERTIES OF THE CLUSTER GALAXIES}

In this section we present the results for the dynamical properties of the galaxies, namely the galaxy number density, velocity anisotropy, and radial velocity dispersion, all derived from our simultaneous analysis of the galaxy surface number density and velocity dispersion data sets. As stated in the previous section, we employed the Jeans equation and used the mass profile we had previously derived from lensing and X-ray data (with our model-independent method described in L08). We present here the results both from fitting simple analytical forms to the dynamical data and from our model-independent approach.

Using the assumed profiles, the simultaneous fit to the two data sets was very good, as expressed by the low $\chi_{r}^{2}=$ $15.6 /(30-5)$, where there are 30 total data points and 5 parameters (from Equations (6) and (7)). The contribution of each data set within the full simultaneous fit to both, was $\chi_{r}^{2}($ number $)=12 /(20-3)$ and $\chi_{r}^{2}($ velocities $)=3.6 /(10-2)$. The fits are shown in Figure 4 . The best-fit parameters of the analytical expressions for the galaxy number density and velocity anisotropy profiles are given in Table 1 .

The derived three-dimensional profile of galaxy number density is shown in Figure 5. The analytical cored profile is consistent with our best-fit model-independent profile. We also tried a cuspy profile as an analytical form for the galaxy number density, instead of a cored profile. Specifically, we adopted a 


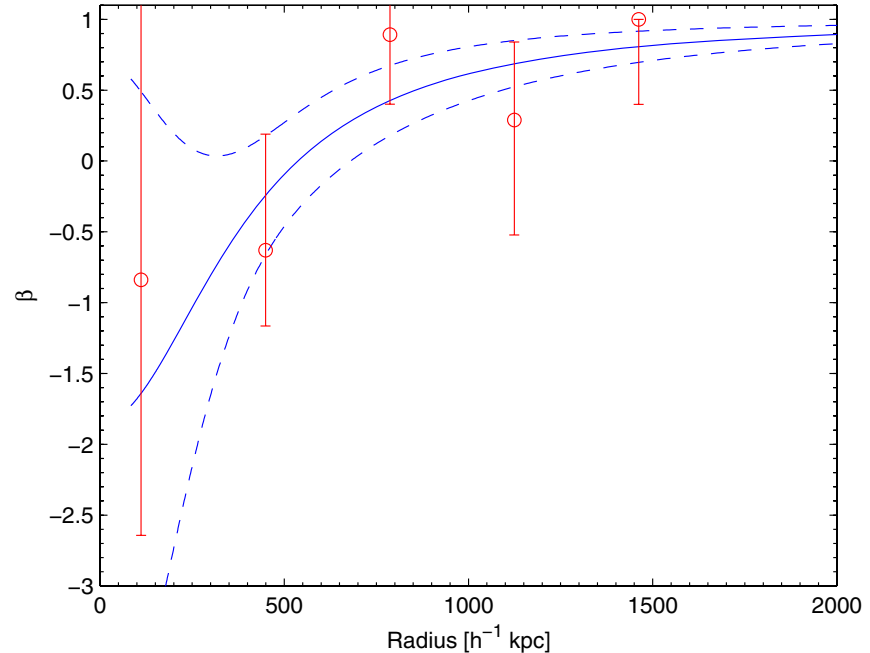

Figure 6. Reconstructed three-dimensional velocity anisotropy profile. We show the best-fit result and $1 \sigma$ range from fitting the simple profile of Equation (7) (solid and dashed curves), where the best-fit model parameters are listed in Table 1. Also shown are the values and $1 \sigma$ errors as derived from the modelindependent approach, where we used five free parameters for the values of the galaxy number density and five (red circles) free parameters for the values of $\beta$ at five equally spaced radii. The value of the velocity anisotropy was extrapolated as constant beyond the last data point.

(A color version of this figure is available in the online journal.)

general form with a $1 / r$ cusp, $n_{\text {gal }}(r)=n_{\text {gal }}(0) /\left[\left(r / r_{s}\right)(1+\right.$ $\left.\left(r / r_{s}\right)\right)^{p}$, which includes NFW $(p=2)$ and Hernquist (1990) $(p=3)$ profiles as special cases. Although this profile gave an acceptable fit, $\chi_{r}^{2}=26.3 /(30-5)$, the fit was worse than that of the cored profile. The best-fit model gave $n_{\text {gal }}(0)=18.8$ and an unrealistic value for $r_{s}, r_{s}=15 h^{-1} \mathrm{Mpc}$; with this value of $r_{s}$ and the deduced $p, p=18.1$, the $p$-dependent term is negligible (implying that only the ratio $n_{\text {gal }}(0) / r_{s}$ is relevant).

The velocity anisotropy profile $\beta$ is shown in Figure 6. Again, the result from fitting a particular simple analytical form for the profile agrees with the result of the model-independent approach which does not assume any particular shape for the profile. While the uncertainties are large, the figure indicates a clear tendency of an increasing $\beta$ with radius. The $\sigma_{r}$ profile, as obtained from the Jeans equation (Equation (2)), is shown in Figure 7.

The analytical expression we used for $\beta$, Equation (7), constrains $\beta$ to equal unity at large radii, corresponding to purely radial orbits. D99 analyzed $N$-body simulations and derived values somewhat smaller than 1 at their limiting radius, $6 r_{200}$. To allow for deviation from unity we also fit the expression $(C+a) \frac{\left(r / r_{c}\right)^{2}}{\left(r / r_{c}\right)^{2}+1}-C$, where $a$ is a free parameter which governs the asymptotic behavior of $\beta$. The value of $a=1$ gave the best fit, also consistent with the value obtained by the modelindependent method at the largest radial point.

\section{DERIVING THE MASS PROFILE}

In Section 4 we used the mass profile as derived from X-ray and lensing data together with the new data on the projected velocity dispersion and the galaxy surface number density to derive the three-dimensional profiles of the velocity anisotropy and galaxy number density. Alternatively, there are at least two different ways to derive the total mass profile of the cluster directly from the data sets on galaxy dynamics. Perhaps the simplest approach is to use only the velocity caustics derived

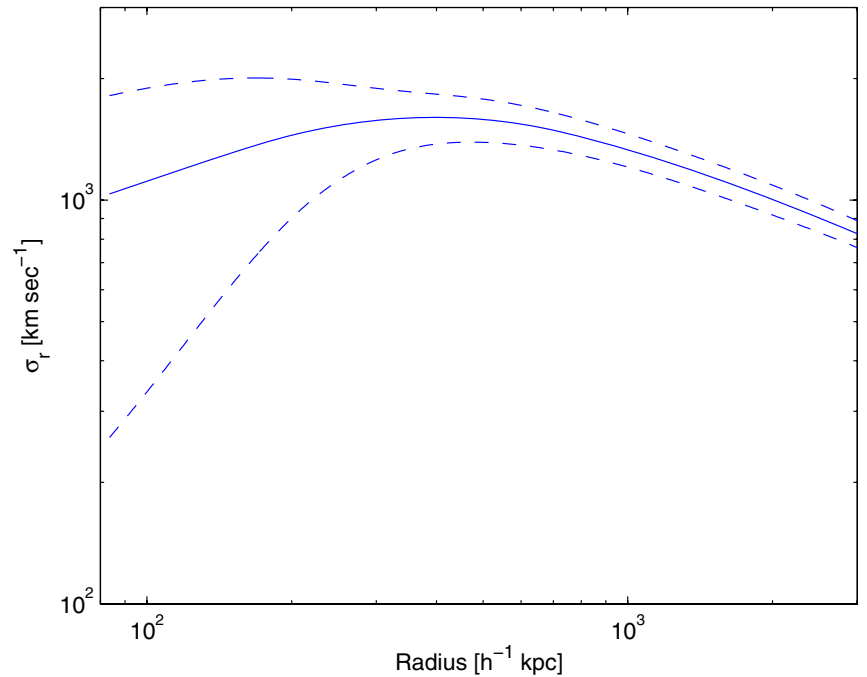

Figure 7. Reconstructed profile of the radial velocity dispersion $\sigma_{r}$. We show the best-fit values and $1 \sigma$ uncertainties from fitting with the simple analytical expressions in Equations (6) and (7) (solid and dashed curves).

(A color version of this figure is available in the online journal.)

above, the amplitude of which is related to the escape velocity, which is a tracer of the cluster mass profile. The second approach is to use both data sets (of the projected velocity dispersion and galaxy surface number density) together with the Jeans equation to fit an NFW mass profile. Thus, we have two independent methods for estimating the cluster mass profile.

\subsection{Mass Profile from Velocity Caustics}

As mentioned already, D99 has shown that the threedimensional mass profile can be fairly well estimated directly from the amplitude of the velocity caustics. In particular, the total mass within radius $r$ is estimated as

$$
M(\leqslant r)=\frac{1}{2 G} \int_{0}^{r} A^{2}(R) d R .
$$

This equation essentially estimates the mass profile based on the local escape velocity, but note that the prefactor of 0.5 is not the result of an exact derivation. This prefactor comes from the more general suggestion by Diaferio \& Geller (1997; hereafter DG97) for the relation between $A$ and $M: M(\leqslant r)=$ $\frac{1}{G} \int_{0}^{r} A^{2}(R) F_{\beta}(R) d R$ in terms of

$$
F_{\beta}(r)=-2 \pi G \frac{\rho(r) r^{2}}{\phi(r)} \frac{3-2 \beta(r)}{1-\beta(r)}
$$

where $\rho(r)$ is the cluster mass density and $\phi(r)=-\frac{G M(<r)}{r}-$ $4 \pi \int_{r}^{\infty} \rho(x) x d x$ is the gravitational potential generated by the cluster. D99 noted that the function $F_{\beta}(r)$ is slowly varying at large radii, $r \gtrsim r_{200} / 3$ (D99), when assuming the NFW profile for the cluster mass density and computed the anisotropy profile $\beta(r)$ from simulations. D99 followed DG97 and set $F_{\beta}$ to be 0.5 , finding that the resulting caustic method recovers the actual cluster mass within a factor of 2 at large radii, $r \sim(0.3-6) r_{200}$, at least with the low concentration parameters of his simulated clusters. Figure 8 shows that for A1689 there is a reasonably good agreement between the mass profile derived from the caustics and that derived from our earlier joint lensing and Xray analysis, except (for the uncorrected caustic method) in the inner region, although the data points even in this region are 


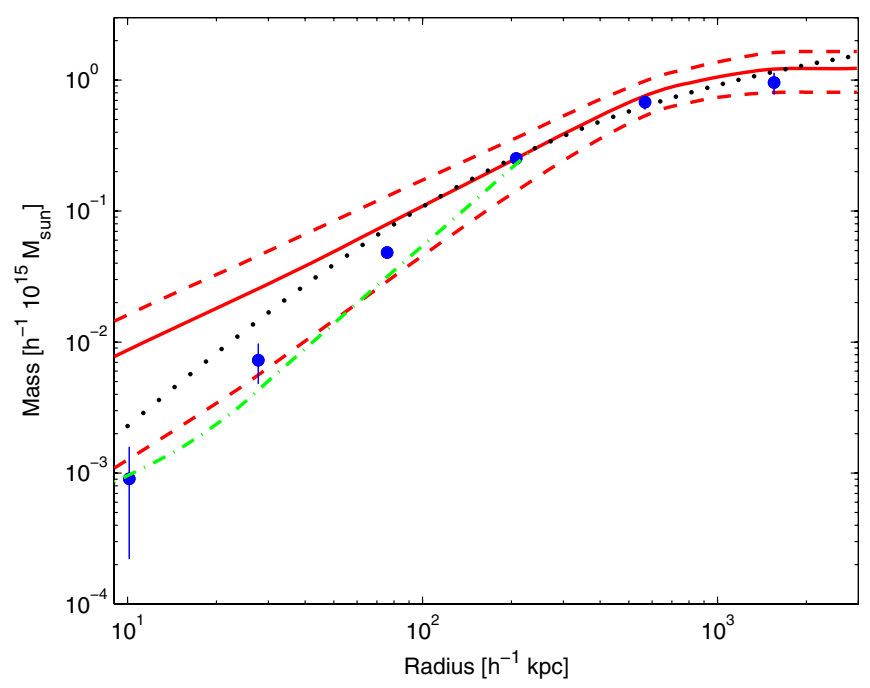

Figure 8. Profile of total enclosed mass, as derived from the caustics (red curve), caustics with $F_{\beta}$ corrected for $r<250 h^{-1} \mathrm{kpc}$ (green dash-dotted curve), lensing and X-ray data (blue circles, L08), and an NFW mass profile fit to the galaxy surface number density and projected velocity dispersion data sets (black dots). The uncertainties (error bars for the lensing and X-ray data based points, and red dashed curves for the caustic based profile) are $1 \sigma$.

(A color version of this figure is available in the online journal.)

within the $1 \sigma$ range of uncertainty. The difference is well below a factor of 2 in the range $\sim(0.1-1.5) h^{-1} \mathrm{Mpc}$. This suggests that the assumption of $F_{\beta}=0.5$ is reasonable for this cluster over a broad radial range. Note that the NFW profile fitted to this cluster gives a relatively high concentration parameter (see Section 3).

At smaller radii, $r \lesssim 0.1 h^{-1} \mathrm{Mpc}$, the two mass profiles differ by $\sim 1 \sigma$. This difference can arise if $F_{\beta}$ is significantly different from 0.5. As a consistency check, we can use the velocity anisotropy profile and the mass profile of the cluster that we derived from the lensing and X-ray analysis to calculate the $F_{\beta}$ profile. Figure 9 shows that at small radii $\left(r<0.1 h^{-1} \mathrm{Mpc}\right)$ $F_{\beta}$ is indeed well below 0.5 . In Figure 9 we compare the $F_{\beta}$ profile derived by our model-independent method to that derived from assuming an NFW profile for the total mass density. The profile determined by D99 from simulations is somewhere in between our two measured profiles: the simulated $F_{\beta}$ increases with radius and then flattens, but only at large radii $\left(\sim \frac{1}{2} r_{\text {vir }}\right)$. Note that in the model-independent method, the point at the largest radius is lower than in the NFW case, since the density there is lower than derived using the NFW model due to the fact that the slope of the total density at large radii for A1689 is slightly steeper than for the NFW profile (B05a; L08). In Figure 8 we also plot the mass profile based on the caustics method when we use the more realistic $F_{\beta}$ value (instead of 0.5 ) at $r<250 h^{-1} \mathrm{kpc}$. The correction improves the agreement between the caustics-based mass profile and the profile derived from the joint lensing and X-ray analysis of L08.

The mass of the cluster derived by the caustics method is roughly equal to the virial mass, $M_{\text {vir }}=(1.23 \pm 0.42) \times$ $10^{15} h^{-1} M_{\odot}$, since the caustics end at about the virial radius (see Section 6).

\subsection{Mass Profile from the Jeans Equation}

Analysis of the lensing data suggests that A1689 has a high concentration parameter (B05a; L08). We can independently check the concentration parameter and the virial mass as

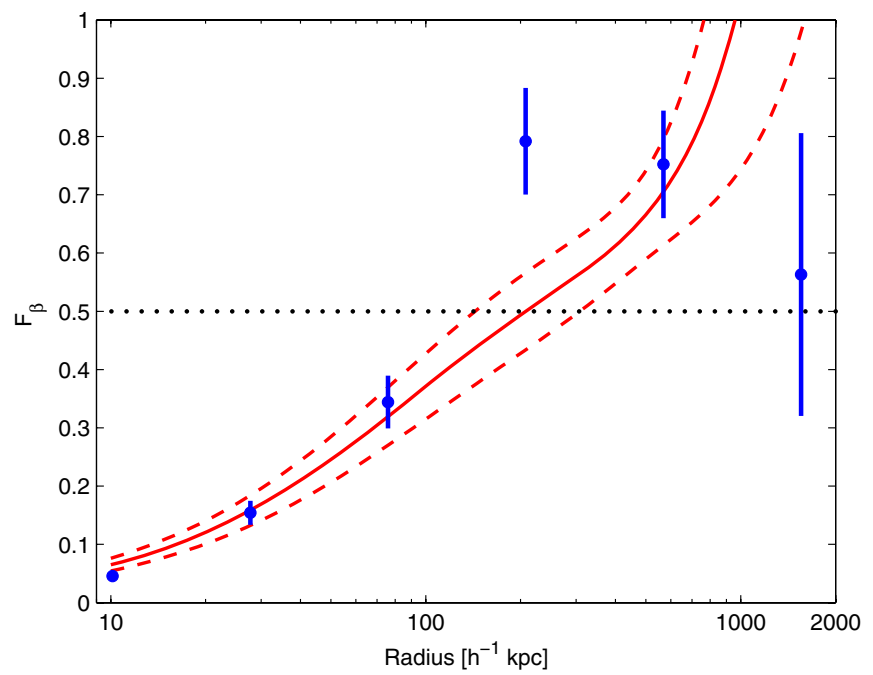

Figure 9. Reconstructed profile of the dimensionless factor $F_{\beta}$ given by Equation (9). We compare two profiles, where we use the total mass density derived by the model-independent method in L08 (blue points) or the best-fit NFW profile from L08 (red curve). The uncertainties (blue error bars for the model-independent method and dashed curves for the NFW model) are $1 \sigma$. Also shown for comparison is the $F_{\beta}=0.5$ (dotted horizontal) line.

(A color version of this figure is available in the online journal.)

derived solely from our two data sets that measure the galaxy surface number density and projected velocity dispersion. A similar attempt to do so was made by Łokas et al. (2006) but with significantly less extensive projected velocity data (see Section 2.2). In addition to what has been done in Section 3, we have also obtained the mass by implementing the following procedure: We use the galaxy number density and velocity anisotropy profiles (Equations (6) and (7)), along with an NFW profile for $M(r)$, which adds free parameters $c_{\text {vir }}$ (the concentration parameter) and $M_{\text {vir }}$ (the virial mass), and derive the parameters of the three profiles by simultaneously fitting to the galaxy number density and velocity data, using the Jeans equation. This method results in relatively weak constraints, as is clear from the rather wide $1 \sigma$ contours shown in Figure 10 (black contours); the deduced mass and concentration are $M_{\text {vir }}=1.6_{-0.8}^{+1.1} \times 10^{15} h^{-1}$, and $c_{\text {vir }}>5.8$ (with no useful upper limit).

The latter method of estimating the cluster mass, from the Jeans equation with assumed profiles, is less precise than the other methods, yielding a partial degeneracy between $c_{\text {vir }}$ and $M_{\text {vir }}$ as shown in Figure 10. We thus expect this constraint to be weak. The observed galaxy number density essentially determines $n_{\text {gal }}(r)$ through Equation (4), and the observed projected velocity dispersion determines a degenerate combination of $\sigma_{r}^{2}$ and $\beta$ at each radius through Equation (5). This yields a degeneracy where for any assumed $\beta(r)$, the Jeans equation yields an $M(r)$ that is consistent with the galaxy dynamical data. In the actual fitting though, this degeneracy is partially broken by the strict analytical forms assumed for the various input profiles. Figure 8 shows that the best-fit NFW mass profile from the Jeans equation is in good agreement with the mass profiles from the other methods.

To get more useful constraints on $c_{\text {vir }}$ and $M_{\text {vir }}$, we combine the two dynamical methods, i.e., the caustics and the Jeans equation. From the caustics method we take only the constraint on $M_{\text {vir }}$, since the mass profile at small radii is uncertain in this method due to the breakdown of the $F_{\beta}=0.5$ assumption (see 


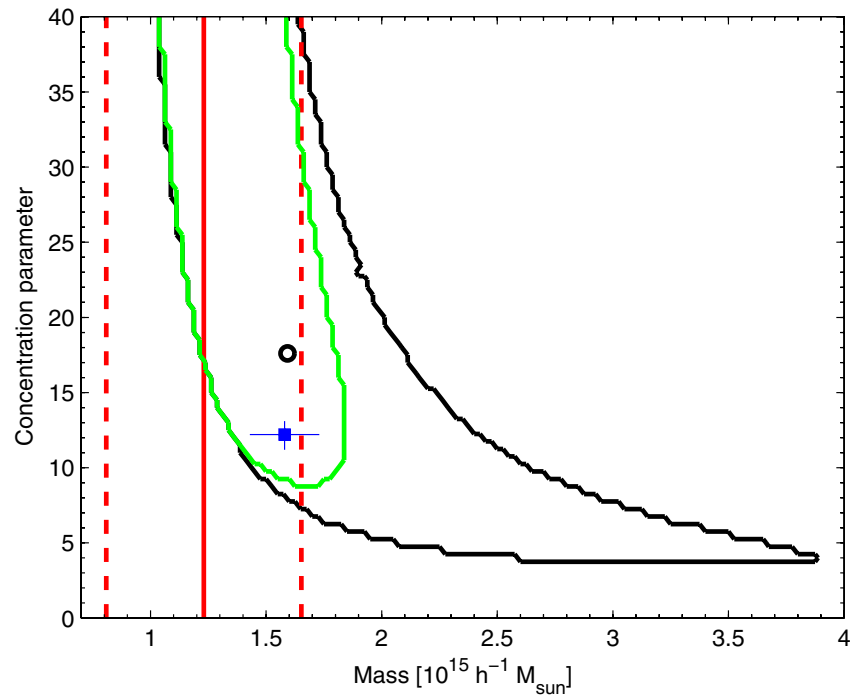

Figure 10. Virial mass and concentration parameter, $c_{\mathrm{vir}}$, derived from NFW fits to the cluster total mass profile. We show the results of an NFW fit to the galaxy dynamics using the Jeans equation (black circle for the best-fit values and black contour for the $1 \sigma$ uncertainty), the mass measured at the virial radius from caustics (red solid and dashed lines for the best-fit value and $1 \sigma$ upper limit), and the joint $1 \sigma$ uncertainty taking both methods, using the Jeans equation and the caustics, under consideration (green contours). For comparison we show the result from the lensing and X-ray data (blue square with $1 \sigma$ error bars; L08).

(A color version of this figure is available in the online journal.)

discussion in the previous subsection). Figure 10 shows that the combined constraints (green contours) are stronger and in good agreement with the values derived from the lensing and X-ray data by L08 (who assumed in this particular analysis an NFW profile for the total mass density and a double beta model for the gas mass density profile). In particular, the combined dynamical methods yield $1 \sigma$ limits of $M_{\mathrm{vir}}=(1.3 \pm 0.4) \times 10^{15} h^{-1} M_{\odot}$ and $c_{\mathrm{vir}}>13.4$.

\section{THE EDGE OF THE CLUSTER}

In this section we summarize various ways of defining a limiting radius for A1689. One way to define the edge of the cluster is to use the observed galaxy number density profile. The fits that we have used in Equations (1) and (6) do not have a sharp cutoff in the number density of galaxies. We thus define an edge as the radius where we can no longer detect cluster members above the contribution of the (noncluster) background galaxy level. For A1689 this point is visible in Figure 1. Specifically, the cluster radial edge was estimated to be where $\Sigma_{\mathrm{gal}} / C_{\mathrm{bg}}>\Delta C_{\mathrm{bg}} / C_{\mathrm{bg}}$ and $\Sigma_{\mathrm{gal}} / C_{\mathrm{bg}}<\Delta C_{\mathrm{bg}} / C_{\mathrm{bg}}$ including uncertainties in $\Sigma_{\mathrm{gal}} / C_{\mathrm{bg}}$, yielding a limiting radius of $2.1_{-0.7}^{+0.8} h^{-1} \mathrm{Mpc}$, where the $1 \sigma$ uncertainties account also for the errors in the various fitting parameters in Equation (1).

Independently, the velocity caustic fits to the projected velocity dispersion data shown in Figure 2 yield a very similar value for the limiting radius, $2.12 \pm 0.07 h^{-1} \mathrm{Mpc}$, where the error includes an estimate of the effect of Poisson noise in the observed number of galaxies. We caution that in simulations the caustics often flatten but do not reach zero at the virial radius; also, the shape of the caustics is somewhat dependent on the particular line of sight (D99). However, the caustics are generally more cleanly defined in data on real clusters than in $N$-body simulations (Rines et al. 2003).

Both of these methods yield a cluster edge limiting radius of $\sim 2 h^{-1}$ Mpc. A similar value was also independently derived from our lensing and X-ray analysis, which depends mostly on the projected DM distribution. We found the virial radius to be $2.14_{-0.29}^{+0.27} h^{-1} \mathrm{Mpc}$ (L08). We conclude that all these different data sets agree reasonably well both in terms of the virial mass and mass profile and in the values they yield for the cluster's limiting radius.

The various ways of defining a limiting radius for A1689 are consistent with each other. This suggests that there is no major infall of DM and galaxies. Significant ongoing infall would add to the projected profiles of mass and galaxy number and also affect the dynamical measurements, likely making the cluster edge less apparent.

\section{DISCUSSION}

In this paper we have continued our exploration of A1689 making use of many high quality data sets available for this cluster. This work builds upon our earlier work on this cluster (L08), where we developed a comprehensive joint analysis of high quality strong lensing (HST/ACS), weak lensing (Subaru), and X-ray (Chandra) measurements, from which we tested the consistency of X-ray and lensing data in a model independent way and derived an improved mass profile for A1689. In this paper we have incorporated two other high quality data sets, the galaxy surface number density measured from deep, wide-field imaging with Subaru/Suprime-Cam and a large spectroscopic study of the internal galaxy dynamics measured using VLT/VIMOS. While the lensing and X-ray data gave us the information on the DM and gas content of the cluster, the two new data sets added here provide direct information on the galaxy distribution and motions, leading to new determinations of the DM distribution of the cluster.

The three-dimensional galaxy number density profile derived from our combined analysis of the above data sets is more consistent with a cored profile, rather than a cuspy profile such as the NFW profile, which seems to fit well the DM density distribution. This is in agreement with Adami et al. (1998) who examined a sample of 62 clusters and found that the majority are better fit with a core than a cuspy profile, though for individual clusters the preference for a cored profile is rarely significant at the $90 \%$ confidence level. The galaxy distribution also resembles a King (1962) profile and falls off as $r^{-3.18 \pm 0.42}$, exceeding the slope of $r^{-2.4 \pm 0.2}$ suggested by Bahcall \& Lubin (1994) in order to explain the " $\beta$-discrepancy." This asymptotic behavior of the cluster galaxy profile at large $r$ is in fact very similar to our total matter profile (dominated by DM), which we have shown can be well fit by an NFW profile where the asymptotic behavior is $r^{-3}$.

A principal finding of our work is the first direct determination of the velocity anisotropy profile for a galaxy cluster. This followed from an application of the Jeans equation, using as input the observed projected velocity dispersion profile, the observed projected galaxy distribution, and our independently determined mass profile, allowing us to solve for the three-dimensional velocity anisotropy as a function of radius. The resulting anisotropy profile is well fit by the expression in Equation (7) proposed by Carlberg et al. (1997) on the basis of $N$-body simulations. The simulations covered a wide range of cosmologies and showed that the radial dependence of the velocity anisotropy $\beta$ has a nearly universal form (Cole \& Lacey 1996; Carlberg et al. 1997), with a characteristic radial dependence. This dependence is also observed in A1689-mainly radial motion is deduced at large radius, tending towards isotropic (or possibly tangential) motion within 
the central region $r \lesssim 500 h^{-1} \mathrm{kpc}$. This presumably is a manifestation of the overall formation and growth of clusters, with initial collapse and virialization of the central region, and continued growth of the cluster mass through accretion, a two-stage process for which there seems to be some evidence also from the DM entropy distribution (e.g., Lapi \& Cavaliere 2009).

Additionally, we used new extensive measurements of galaxy positions and velocities to determine the cluster mass profile. This was done in two independent ways; first, using the velocity data alone, we identified clearly apparent velocity caustics using the method of D99. The derived amplitude of the velocity caustics was interpreted as the local escape velocity, from which the mass profile was determined using Equation (8). Secondly, we followed the traditional approach of using the Jeans equation (Equation (2)), incorporating both the galaxy surface number density and the projected velocity data, and adopting the above velocity anisotropy profile. These two different mass estimates are in good agreement with the profile derived from our earlier lensing and X-ray analysis (L08), as shown in Figure 8.

In estimating the caustic-based mass profile we adopted the previously suggested value $F_{\beta}=0.5$. We were able to separately check this assumption using the velocity anisotropy profile obtained as described above. Our previously determined mass profile made it possible to deduce $\beta$; the comparison of the resulting mass profile with that from our previous lensing/ $\mathrm{X}$-ray analysis is then essentially a consistency check on the general validity of the caustic method. We found that at large radii, $r \gtrsim 100 h^{-1} \mathrm{kpc}$, the factor $F_{\beta}$ varies slowly with radius and stays within $\sim 50 \%$ of the value of 0.5 (see Figure 9), so that the relatively simple caustic method yields the mass profile accurately except at the center. Note that D99 derived the mean value of $F_{\beta}=0.5$ from simulated CDM halos, which typically have concentration parameter $\lesssim 7$, well below that of A1689 $\left(c_{\text {vir }}=12.2_{-1}^{+0.9} ;\right.$ L08). Thus, our results suggest that the causticbased mass estimation is applicable also for high concentration clusters. This shows that dynamical analysis may be improved upon by combining the traditional method based on the Jeans equation with the additional insight gained from the caustics (in relaxed clusters).

The virial mass derived from the caustic method, $M_{\text {vir }}=$ $(1.23 \pm 0.42) \times 10^{15} h^{-1} M_{\odot}$, and from the Jeans equation, $M_{\text {vir }}=1.6_{-0.8}^{+1.1} \times 10^{15} h^{-1} M_{\odot}$. The two combined dynamical methods gave $M_{\text {vir }}=(1.3 \pm 0.4) \times 10^{15} h^{-1} M_{\odot}$, as compared with the value obtained in our joint lensing/X-ray analysis (L08), $M_{\text {vir }}=(1.58 \pm 0.15) \times 10^{15} h^{-1} M_{\odot}$. These mass estimates are consistent, and in agreement also with the result of Umetsu \& Broadhurst (2008), who combined strong lensing, weak lensing distortion and magnification data in a twodimensional analysis (without assuming axial symmetry), and derived $M_{\text {vir }}=1.5_{-0.3}^{+0.6} \times 10^{15} h^{-1} M_{\odot}$ (where this $1 \sigma$ error includes both statistical and systematic uncertainties).

A novel aspect of our work is an estimation of the limiting radius of A1689 in several different ways. All our data sets independently indicate that this cluster has a relatively well defined edge radius of $\sim 2.1 h^{-1}$ Mpc. We see this in terms of the galaxy distribution which is flat beyond this radius, indicating that the galaxy number density reached the background level, from the velocity caustics which attain (practically) zero velocity at this radius, and also from the virial radius derived from the best-fit NFW profile. This interesting agreement should be checked in other massive clusters. In principle, we may expect a clear signature of the virial radius in the absence of significant ongoing infall of galaxies and mass onto the cluster.

Massive clusters $\left(M \sim 10^{15} h^{-1} M_{\odot}\right)$ are theoretically expected to have relatively low mass concentrations, with $c_{\text {vir }}(z=0) \sim 5$, as determined from cosmological simulations of the standard $\Lambda \mathrm{CDM}$ (Neto et al. 2007; Hennawi et al. 2007; Duffy et al. 2008). However, significantly higher values, $c_{\text {vir }} \sim 10-15$, were deduced from detailed weak and strong lensing measurements of several well studied massive clusters (Kneib et al. 2003; Gavazzi et al. 2003; B05a; Kling et al. 2005; Limousin et al. 2007; L08; Umetsu \& Broadhurst 2008; Broadhurst et al. 2008). This discrepancy can also be expressed in terms of the Einstein radius, which provides a modelindependent measure of the central mass within clusters, and can be compared with the total (virial) mass to get a measure of the degree of concentration. The observed Einstein radii of many massive clusters lie in the range $20^{\prime \prime}-50^{\prime \prime}$, around twice the expected range after allowance for lensing and projection biases (Broadhurst \& Barkana 2008). In addition, Sadeh \& Rephaeli (2008) showed that even considering the full probability distribution function of halo formation times does not fully remove the discrepancy.

While we have not measured the concentration parameter of A1689 as accurately as from the lensing data, we have confirmed its high value. The two combined dynamical methods yield $c_{\mathrm{vir}}>13.4$ (at $1 \sigma$ confidence), consistent with $c_{\mathrm{vir}}=12.2_{+0.9}^{-1}$ derived in our joint lensing and X-ray analysis (L08), and with $c_{\text {vir }}=12.7 \pm 3$ from Umetsu \& Broadhurst (2008). Clearly, this comparison should be examined for other well studied clusters, but at least in A1689 this independent dynamical measurement, which is less susceptible to projection bias than lensing, is in good agreement with the lensing results, and can be seen (at least partly) as evidence for the validity of (the assumption of) spherical symmetry.

The effect of triaxiality may significantly lower the observationally deduced concentration parameter (Oguri et al. 2005; Gavazzi 2005). An independent analysis of A1689 by Corless et al. (2009) does not find this to be significant, $c_{\mathrm{vir}}=12.2 \pm 6.7$, in good agreement with our earlier analysis, where the sizable uncertainty reflects the unknown triaxiality and the relatively shallow imaging used. An important step in empirically examining the effect of triaxiality and geometric projection bias is to see whether there is any systematic difference between the distribution of mass profiles derived for clusters selected by different methods. In a recent analysis of a cluster sample selected using weak lensing, Hamana et al. (2008) — who used the sample of Miyazaki et al. (2002) — did not find evidence for selection bias in mass estimates between clusters selected by weak lensing as compared with other selection methods, such as optically or by X-ray emission. Also, Duffy et al. (2008) found that the derived concentrations for X-ray selected samples taken from the literature lie well above the expected values based on cosmological $\mathrm{N}$-body simulations with the most recent cosmological parameters.

At least some of the high deduced values of $c_{\mathrm{vir}}$ are expected in early dark energy (EDE) models. In these models there is a non- negligible dark energy component even at high redshift, in contrast with the standard model; consequently, clusters form earlier and are more abundant than predicted in $\Lambda$ CDM (e.g, Sadeh \& Rephaeli 2008). In recent work using $\mathrm{N}$-body simulations it was found that halos in an EDE model show higher concentrations at a given halo mass (Grossi \& Springel 2009). Interestingly, in contrast with expectations from 
semi-analytic approaches, recent simulations show that EDE does not significantly affect the statistics of nonlinear structures, at least at low redshifts (Francis et al. 2009; Grossi \& Springel 2009). In addition, the measured X-ray background cannot rule out EDE models if their normalization is defined by the halo density at $z=0$ (Lemze et al. 2009). We intend to explore further the issue of values of $c_{\text {vir }}$ by extending our dynamical work to additional well studied clusters for which we have obtained precisely measured lensing-based mass profiles.

We thank Oliver Czoske for providing the redshift survey information for A1689 and for many helpful discussions. We also acknowledge discussions with Sharon Sadeh, Antonaldo Diaferio, Masataka Fukugita, Hagai Netzer, and the referee for useful comments. We wish to thank the referee for a thorough reading of a previous version of the paper, and for several useful suggestions. This paper was partly written while D.L. and T.B. were visitors of the Institute for the Physics and Mathematics of the Universe, University of Tokyo, which we thank for its generous hospitality. R.B. is grateful for support from the ICRR in Tokyo, Japan, the Moore Distinguished Scholar program at Caltech, and the John Simon Guggenheim Memorial Foundation. K.U. is partially supported by the National Science Council of Taiwan under the grant NSC97-2112-M-001-020MY3.

\section{REFERENCES}

Adami, C., Mazure, A., Katgert, P., \& Biviano, A. 1998, A\&A, 336, 63

Andersson, K. E., \& Madejski, G. M. 2004, ApJ, 607, 190

Bahcall, N. A., \& Lubin, L. M. 1994, ApJ, 426, 513

Balogh, M. L., Couch, W. J., Smail, I., Bower, R. G., \& Glazebrook, K 2002, MNRAS, 335, 10

Benatov, L., Rines, K., Natarajan, P., Kravtsov, A., \& Nagai, D. 2006, MNRAS, 370,427

Binney, J., \& Tremaine, S. 1987, in Galactic Dynamics, ed. J. P. Ostriker (Princeton, NJ: Princeton Univ. Press), 747

Biviano, A., \& Girardi, M. 2003, ApJ, 585, 205

Biviano, A., \& Katgert, P. 2004, A\&A, 424, 779

Bradač, M., et al. 2006, ApJ, 652, 937

Broadhurst, T., Takada, M., Umetsu, K., Kong, X., Arimoto, N., Chiba, M., \& Futamase, T. 2005, ApJ, 619, L143 (B05a)

Broadhurst, T., et al. 2005, ApJ, 621, 53

Broadhurst, T., Umetsu, K., Medezinski, E., Oguri, M., \& Rephaeli, Y. 2008, ApJ, 685, L9

Broadhurst, T. J., \& Barkana, R. 2008, MNRAS, 390, 1647

Bower, R. G., Benson, A. J., Malbon, R., Helly, J. C., Frenk, C. S., Baugh, C. M., Cole, S., \& Lacey, C. G. 2006, MNRAS, 370, 645

Carlberg, R. G., et al. 1997, ApJ, 485, L13

Clowe, D., Gonzalez, A., \& Markevitch, M. 2004, ApJ, 604, 596

Cole, S., \& Lacey, C. 1996, MNRAS, 281, 716

Corless, V. L., King, L. J., \& Clowe, D. 2009, MNRAS, 393, 1235

Czoske, O. 2004, in IAU Colloq. 195, Outskirts of Galaxy Clusters: Intense Life in the Suburbs, ed. A. Diaferio (Cambridge: Cambridge Univ. Press), 183

Danese, L., de Zotti, G., \& di Tullio, G. 1980, A\&A, 82, 322

den Hartog, R., \& Katgert, P. 1996, MNRAS, 279, 349

Diaferio, A., \& Geller, M. J. 1997, ApJ, 481, 633 (DG97)

Diaferio, A. 1999, MNRAS, 309, 610 (D99)

Diaferio, A., Geller, M. J., \& Rines, K. J. 2005, ApJ, 628, L97

Diemand, J., Moore, B., \& Stadel, J. 2004, MNRAS, 352, 535

Duc, P.-A., et al. 2002, A\&A, 382, 60
Duffy, A. R., Schaye, J., Kay, S. T., \& Dalla Vecchia, C. 2008, MNRAS, 390, L64

Francis, M. J., Lewis, G. F., \& Linder, E. V. 2009, MNRAS, 394, 605

Fuchs, B., \& Materne, J. 1982, A\&A, 113, 85

Gavazzi, R., Fort, B., Mellier, Y., Pelló, R., \& Dantel-Fort, M. 2003, A\&A, 403, 11

Gavazzi, R. 2005, A\&A, 443, 793

Geller, M. J., Diaferio, A., \& Kurtz, M. J. 1999, ApJ, 517, L23

Girardi, M., Giuricin, G., Mardirossian, F., Mezzetti, M., \& Boschin, W. 1998, ApJ, 505, 74

Grossi, M., \& Springel, V. 2009, MNRAS, 394, 1559

Hamana, T., Miyazaki, S., Kashikawa, N., Ellis, R. S., Massey, R. J., Refregier, A., \& Taylor, J. E. 2008, arXiv:0808.3813

Hennawi, J. F., Dalal, N., Bode, P., \& Ostriker, J. P. 2007, ApJ, 654, 714

Hernquist, L. 1990, ApJ, 356, 359

Hwang, H. S., \& Lee, M. G. 2008, ApJ, 676, 218

Kaiser, N. 1987, MNRAS, 227, 1

Katgert, P., Biviano, A., \& Mazure, A. 2004, ApJ, 600, 657

Kawahara, H., Suto, Y., Kitayama, T., Sasaki, S., Shimizu, M., Rasia, E., \& Dolag, K. 2007, ApJ, 659, 257

Kazantzidis, S., Magorrian, J., \& Moore, B. 2004, ApJ, 601, 37

King, I. 1962, AJ, 67, 471

Kling, T. P., Dell'Antonio, I., Wittman, D., \& Tyson, J. A. 2005, ApJ, 625, 643

Kneib, J.-P., et al. 2003, ApJ, 598, 804

Lapi, A., \& Cavaliere, A. 2009, ApJ, 692, 174

Lemze, D., Barkana, R., Broadhurst, T. J., \& Rephaeli, Y. 2008, MNRAS, 386, 1092 (L08)

Lemze, D., Sadeh, S., \& Rephaeli, Y. 2009, arXiv:0904.1983

Limousin, M., et al. 2007, ApJ, 668, 643

Łokas, E. L., Prada, F., Wojtak, R., Moles, M., \& Gottlöber, S. 2006, MNRAS 366, L26

Mandelbaum, R., Seljak, U., \& Hirata, C. M. 2008, J. Cosmol. Astropart. Phys., JCAP08(2008)006

Markevitch, M., Gonzalez, A. H., David, L., Vikhlinin, A., Murray, S., Forman, W., Jones, C., \& Tucker, W. 2002, ApJ, 567, L27

Mazzotta, P., Rasia, E., Moscardini, L., \& Tormen, G. 2004, MNRAS, 354, 10

Medezinski, E., et al. 2007, ApJ, 663, 717 (M07)

Miyazaki, S., et al. 2002, ApJ, 580, L97

Navarro, J. F., Frenk, C. S., \& White, S. D. M. 1997, ApJ, 490, 493 (NFW)

Neto, A. F., et al. 2007, MNRAS, 381, 1450

Oguri, M., Takada, M., Umetsu, K., \& Broadhurst, T. 2005, ApJ, 632, 841

Pisani, A. 1993, MNRAS, 265, 706

Pisani, A. 1996, MNRAS, 278, 697

Regos, E., \& Geller, M. J. 1989, AJ, 98, 755

Reisenegger, A., Quintana, H., Carrasco, E. R., \& Maze, J. 2000, AJ, 120, 523

Riemer-Sorensen, S., Paraficz, D., Ferreira, D. D. M., Pedersen, K., Limousin, M., \& Dahle, H. 2009, ApJ, 693, 1570

Rines, K., Geller, M. J., Kurtz, M. J., \& Diaferio, A. 2003, AJ, 126, 2152

Rines, K., \& Diaferio, A. 2006, AJ, 132, 1275

Sadeh, S., \& Rephaeli, Y. 2008, MNRAS, 388, 1759

Sanchis, T., Łokas, E. L., \& Mamon, G. A. 2004, MNRAS, 347, 1198

Sand, D. J., Treu, T., Ellis, R. S., Smith, G. P., \& Kneib, J.-P. 2008, ApJ, 674, 711

Sharples, R. M., Ellis, R. S., \& Gray, P. M. 1988, MNRAS, 231, 479

Silverman, B. W. 1986, Monographs on Statistics and Applied Probability (London: Chapman and Hall)

Spergel, D. N., et al. 2007, ApJS, 170, 377

Teague, P. F., Carter, D., \& Gray, P. M. 1990, ApJS, 72, 715

Tegmark, M., et al. 2004, Phys. Rev. D., 69, 103501

Umetsu, K., \& Broadhurst, T. 2008, ApJ, 684, 177

Vikhlinin, A. 2006, ApJ, 640, 710

Wojtak, R., \& Łokas, E. L. 2007, MNRAS, 377, 843

Wojtak, R., Łokas, E. L., Gottlöber, S., \& Mamon, G. A. 2005, MNRAS, 361, L1

Wojtak, R., Łokas, E. L., Mamon, G. A., Gottlöber, S., Prada, F., \& Moles, M. 2007, A\&A, 466, 437

Xue, S.-J., \& Wu, X.-P. 2002, ApJ, 576, 152 